\title{
Developing institutions for cancer care in low-income and middle-income countries: from cancer units to comprehensive
}

\section{cancer centres}

\author{
Bhawna Sirohi, Kalipso Chalkidou, C S Pramesh, Benjamin O Anderson, Patrick Loeher, Omar El Dewachi, Omar Shamieh, Shailesh V Shrikhande, \\ R Venkataramanan, Groesbeck Parham, Mulindi Mwanahamuntu, Tim Eden, Audrey Tsunoda, Arnie Purushotham, Susannah Stanway, \\ Goura K Rath, Richard Sullivan
}

Global cancer centres operate across different sizes, scales, and ecosystems. Understanding the essential aspects of the creation, organisation, accreditation, and activities within these settings is crucial for developing an affordable, equitable, and quality cancer care, research, and education system. Robust guidelines are scarce for cancer units, cancer centres, and comprehensive cancer centres in low-income and middle-income countries. However, some robust examples of the delivery of complex cancer care in centres in emerging economies are available. Although it is impossible to create an optimal system to fit the unique needs of all countries for the delivery of cancer care, we summarise what has been published about the development and management of cancer centres in low-income and middle-income countries so far and highlight the need for clinical and political leadership.

\section{Introduction}

The progress of cancer management has been marked by rapid growth of the research endeavour and the increased need to deliver complex care across different health ecosystems, topographies, and countries. In the past decade, key drivers for improved global cancer research and the delivery of affordable a nd equitable care have been examined. ${ }^{1}$ This effort to identify i ssues, barriers, and solutions that are suitable for global cancer control has mostly focused on specific areas including women's cancers, ${ }^{2}$ surgery, radiotherapy, ${ }^{3,4}$ continents and countries (eg, Africa, ${ }^{5}$ India, ${ }^{6}$ and Latin America ${ }^{7}$ ), and conceptual frameworks (eg, universal health coverage, the so-called grand convergences in health, ${ }^{8}$ and the 25 -by- 25 goals [reduction of premature mortality by $25 \%$ by 2025$]^{9}$ ). Additionally, capacity and capability building (eg, Zambia's progress against cervical cancer $^{10}$ ), innovation and technology, ${ }^{11}$ resource stratification a round models of care, and workforce planning have been popular themes for discussion and analysis. However, the organisational and structural framing-cancer units, cancer centres, and comprehensive cancer centres (panel 1)-have been largely absent from the discussion. In this Review, we explore factors that influence the development of a cancer centre, key experiences, lessons learnt from the development of these centres in specific countries and regions, and challenges in delivering cancer care in diverse situations.

In any policy study, the normative framework for analysis should incorporate both critical and positive components; however, there is a dearth of empirical work on the former. No cancer centre model system has been subjected to any type of in-depth qualitative or quantitative analysis; even peer reviews of cancer centres by supranational bodies (such as WHO and International Atomic Energy Agency), and national systems, do not have transparent methodologies and are unpublished and unverifiable in terms on their effect on cancer care. Even when analysis of cancer centres is reasonably transparent, the effect on outcomes, or indeed any metric of improved cancer control, is absent. Therefore, any critique of individual cancer centres or models for cancer centre development remain highly subjective and so here we focus on a positivist analytical framework.

Global cancer centres operate across a wide range of sizes, scales, and ecosystems. Understanding the essential aspects of the creation, organisation, accreditation, and activities within these settings is crucial for developing an affordable, equitable, and quality cancer care, research, and education system. The growing burden of cancer in low-income and middle-income countries (LMICs), in particular, has led to an opportunistic increase in the number of cancer centres with little or no regulation or transparent national accountability. Any hospital that treats a
Lancet Oncol 2018
19: e395-406
Barts Health NHS Trust, London, UK (B Sirohi FRCP); Imperial College London, London UK
(Prof K Chalkidou MD); Centre for Global Development, London, UK (Prof K Chalkidou); Tata Memorial Centre, Mumbai, India
(Prof C S Pramesh MS Prof SV Shrikhande MS); Fred Hutchinson Cancer Research Center, Seattle, WA, USA (Prof B O Anderson MD); Indiana University Medical Center, Indianapolis, IN, USA (Prof P Loeher MD); Global Health Institute, American University of Beirut, Beirut, Lebanon (Prof O El Dewachi MD); King Hussein Cancer Center Amman, Jordan (Prof O Shamieh MD); Tata Trusts, Mumbai, India (R Venkataraman MSc, Prof A Purushotham MD); University of North Carolina, Chapel Hill, NC, USA (Prof G Parham MD); University of Zambia, Lusaka, Zambia

\section{Panel 1: Definition of cancer centres}

The term cancer centre broadly covers three types of establishment treating patients with cancer, as defined in high-income settings:

- Cancer units are usually dedicated cancer wards within the general hospital setting, including nursing homes and hospices, particularly in low-income and middle-income countries (LMICs)

- Cancer centres are dedicated multispecialty centres that can be stand alone or part of a general hospital complex, including site-specific cancer centres (eg, haemato-oncology, breast, radiotherapy, hepato-pancreato-biliary, and palliative) often referred to as secondary or district cancer centres (as seen in most National Health Service hospitals in the UK)

- Comprehensive cancer centres are the highest tier and cover multidisciplinary centres that might be stand alone or part of general hospital complexes. These centres deliver not only the full range of cancer care, but also prevention, research, training, and education locoregionally or nationally and are often subject to national and international accreditation processes (eg, National Cancer Institute accreditation in the USA or Organisation of European Cancer Institute accreditation across Europe). 
(M Mwanahamuntu); University of Manchester, Manchester, UK Erasto Gaertner, Instituto de Oncologia do Paraná and Universidade Positivo, Curitiba, Brazil (A Tsunoda MD); King's Health Partners Comprehensive Cancer Centre and Institute of Cancer Policy, School of Cancer Sciences, Kings College London, London, UK (Prof R Sullivan MD, Prof A Purushotham); Royal Marsden NHS Foundation Trust, London, UK (S Stanway MD); and All India Institute of Medical Sciences, New Delhi, India (Prof G K Rath MD) Correspondence to: Dr Bhawna Sirohi, Barts Health NHS Trust, Barts Cancer Institute, London EC1A 7BE, UK bhawna.sirohi13@gmail.com (ProfT Eden MD); Hospital

patient with cancer can call itself a comprehensive cancer centre, irrespective of the facilities, qualifications, or expertise that are available to the centre. In this health policy analysis, we examine cancer from a structural and organisational perspective, focusing on the professionalisation of cancer centres as part of the emergence of global attention on all aspects of healthsystem strenghtening. ${ }^{12}$ Figure 1 shows the factors that can influence the development of a cancer centre.

\section{Cancer centre development}

The organisation of cancer care into specific centres happened organically through early developments in cancer therapeutics and the move from cancer as a terminal condition to one that could be treated with curative intent. ${ }^{13}$ Models for cancer care were first developed in the late 1800 s with the creation of specific wards dedicated to cancer in general hospitals. ${ }^{14}$ The increase of research into cancer gave rise to early prototype cancer centres such as the University of Buffalo Pathological Laboratory that was opened in 1898 , which became the National Cancer Institute-designated Roswell Park Cancer Institute in 1992, and Memorial Sloan Kettering Cancer Centre, which opened in 1884 as a New York cancer hospital on Manhattan's Upper West Side. In Europe, the Royal Marsden in the UK, the Institut Gustave Roussey cancer centre in France, Radiumhemmet in Solina, Sweden, and the Netherlands Cancer Institute in Amsterdam, were all opened between the 1920s and the 1950s.

Early development of the cancer centre model also happened in LMICs. In India, the creation of the CSI Medical Mission Hospital, Neyyoor, south India, in 1831, the Tata Memorial Centre, Mumbai, in 1941, and the Cancer Institute (Women's Indian Association), Chennai, in 1950 ushered in a new era of cancer centres across India. In Latin America, some cancer centres rose to early prominence, including the Dr Alrnaldo Cancer Institute, which was opened in 1920, the Radium Institute, Belo Horizonte, in Brazil, which was opened in 1922, and the Cancer Centre of the Federal District, which led to the creation of the Brazilian cancer centre network (Instituto Nacional de Cáncer) in 1937. Other cancer centres that

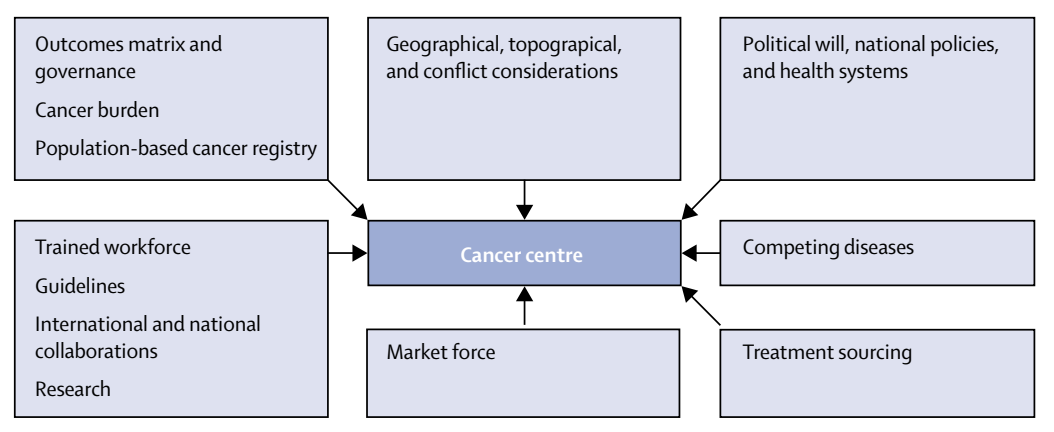

Figure 1: Factors that can influence the development of a cancer centre opened in this period included the National Cancer Institute, Bogotá, Colombia (1934), National Cancer Institute, Lima, Peru (1939), and Mexico City, Mexico (1946). China established their first cancer centre as the Shanghai Cancer Institute (1931), and the Cancer Institute of the Chinese Academy of Medical Sciences, Beijing (1958). In the Middle East, Iran and Egypt led the way with the Cancer Institute, Tehran, Iran (1949) and the National Cancer Institute, Cairo, Egypt (1950).

The formal organisation and accreditation of cancer centres started in the USA with the 1971 National Cancer Act, which led to the creation of the National Cancer Institute and other comprehensive cancer centres for the country. ${ }^{15}$ In other high-income settings, cancer centre development has taken a bottom-up approach. The establishment of Institut National du Cancer in France in the mid-2000s led to the formalisation of cancer centres within national legislation that mandated research and care, as well as budget allocation. Cancer centres in other European Union countries have evolved in a similar way but with different legislative framing and funding. In the past 5 years, the Organisation of European Cancer Institutes, originally founded in 1979, has begun a formal system of cancer centre accreditation for cancer centres in Europe. $^{16}$ Additionally, international accreditation schemes have been developed, some of which are generic (eg, Joint Care Commission), and some of which are specific to cancer (eg, European Society of Medical Oncology designated centres for integrative oncology and palliative care). Various emerging powers have also begun formal processes and networks for the national accreditation of cancer centres-eg, the National Cancer Grid of India. ${ }^{17}$

\section{Cancer centre development in high-income settings \\ Service and research}

The development of cancer centres in high-income settings over the past 50 years has been driven by three key factors: first, the need to provide increased volumes of care with increased quality (eg, in onco-surgery ${ }^{18}$ ) by use of complex models of care; second, the need to deliver affordable, multidisciplinary care with the realisation that major cancer centres provide economies of scale; and third, the rapid emergence of translational cancer research requiring close interactions between laboratories and the clinic. ${ }^{19}$ Central to the delivery of cancer care in high-income countries (HICs) is the complex nature of multidisciplinary treatment and supportive services (coordinators, nurse specialists, patient navigators, pathology, and radiology) within cancer centres. These cancer centres have played an important part in the development of oncology in terms of refining existing treatments (eg, radiotherapy techniques, dosage, regimens, and equipment) and cancer surgery (eg, deescalating surgery in breast and rectal cancers for organ 
preservation, endoscopic treatment of early cancers, and surgical oncology techniques), as well as introducing new treatments and de-escalating existing ones (eg, avoiding fluorouracil in breast cancer adjuvant therapy, decreasing the number of cycles of therapy in breast and colon cancer, and refining e ndocrine $t$ herapy) $)^{20,21} \mathrm{t}$ hrough observational studies and clinical trials. These developments can be directly extrapolated to LMICs because the principles of cancer treatment are the same..$^{22}$

Ongoing provision of high care standards is encouraged in HICs by adherence to the principles of clinical governance such as regular clinical audits, patient-public involvement, risk management, education and training of staff, adherence to evidence-based g uidelines, and robust management structures. Collaborations between cancer centres in HICs and those in LMICs have also played a key part in the development of translational and clinical research, including clinical trials. ${ }^{23}$

\section{Education and training}

In HICs, cancer centre development has been concurrent with the development of a professional cancer workforce. Cancer centres have a major role not only as foci for national training programmes but also in training pioneer cancer leaders from LMICs (eg, in childhood cancers $^{24}$ ). Cancer centres in Japan have also had an important influence on the development of cancer centres in LMICs with a specific focus on surgery. ${ }^{25}$ Additionally, health-care professionals from LMICs working in cancer centres in HICs have played a crucial part in the development of partnerships between LMICs and HICs in education and training.

Cancer centres as foci for developing workforce capacity and capability in high-income settings has been balanced by various national policies that encourage out-migration of workers to the private sector, or from rural to urban cancer centres, which leads to cancer centres in rural areas being understaffed. Solutions to this problem have been distinct and heterogeneous but some general lessons can be learned: first, c ancer c entres c an b e the c entral focus for general cancer workforce training; second, national policies are needed to ensure that patients are referred and care is shared between cancer centres; and third, trainees need to be rotated proactively between different cancer centres. ${ }^{26}$

\section{Strategic frameworks}

Cancer centre development has been subject to the unique political economy and health system in each HIC. The formal template for the development and linkage of cancer centres has been led by the USA with its precise description and typologies of what makes a cancer centre comprehensive. ${ }^{27}$ However, this cancer centre model has not been replicable in other systems. The unique socioeconomics of cancer in the USA, coupled with the federal nature of care, its strong private sector, and high amount of research, creates both advantages and disadvantages for this national cancer centre model..$^{28}$ However, the model has been highly effective for the development of cancer leadership globally, particularly to drive research. Crucial issues affecting comprehensive and non-comprehensive cancer centres in the USA are multiple and reflect the tensions and needs that can be found in most high-income settings. Affordability and equity of care are important issues for cancer centre sustainability in high-income settings, which drives improved networking. ${ }^{29}$ The rapid evolution of the research agenda is also creating an environment in which cancer centres are re-appraising their roles and deciding whether the era of population to laboratory research in a single cancer centre is feasible. ${ }^{30}$

Outside the USA, other countries such as France and the $\mathrm{UK}^{26}$ have sought to develop cancer centres within different strategic and legislative frameworks but with an increased emphasis on prevention, survivorship, patient experience, and quality of life after cancer. However, at their core, the research agenda has been similar to that driving cancer centres in the USA, with a substantial focus on translational cancer medicine and pharmaceutical cancer research. Analysis of research activities by site (eg, lung) or domain (eg, surgery) has shown a substantial deficit in the cancer research agenda that has been developed by cancer centres in HICs, particularly in radiotherapy, surgery, palliative care, and supportive care. The influence of political national leadership, philanthropic research funders, and the private sector has narrowed the research spectrum at the same time as many cancer centres are seeing unprecedented increases in research funding.

The global engagement of cancer centres in HICs in cancer centre development in LMICs has been modest. Although some centres have developed formal research and training engagement (eg, Academic Model Providing Access to Healthcare [AMPATH] ${ }^{31}$ and Partners in Health $^{32}$ ), global cancer efforts has remained low on their development agenda. This situation partly reflects the priorities of research funders and national funding for care in HICs and partly a separation of agendas in terms of the development of expensive models of care. Despite these obstacles, cancer centres in HICs have developed some progressive and sustainable twinning models in adult and childhood cancers. ${ }^{23,33}$ The latter has been particularly successful with a combination of childhood cancer centre institutional support and specific research funders. ${ }^{34}$ Many of the global cancer programmes used in cancer centres in HICs have been built on substantial research and programme investments in infectious disease research (eg, in HIV/AIDS), which could offer opportunities for scaling up cancer centres in other settings..$^{31,35}$

Overall, the development of cancer centres and models in HICs provides important lessons for cancer centre development in LMICs including, the delivery of quality optimal care that can be extrapolated to most settings, 
multidisciplinary working, and the use of local data to drive quality improvements. Importantly, each country should develop its own strategic approaches to cancer centre development that fit within their unique historical, social, and economic context.

\section{Cancer centres in LMICs}

Initiatives are being undertaken in LMICs to deliver optimal cancer care by developing cancer centres, but many of these initiatives are currently fragmented and uncoordinated. The challenges of quality, value, affordability, and equality that cancer centres in HICs face are multiplied in LMICs. A further general challenge in cancer centre development is the restriction of funds to support public infrastructure development coupled with the challenges of trying to expand a suitable workforce. Additionally, limitations on research funding have meant that even cancer centres with major clinical and teaching portfolios often produce few research outputs. Even when research activity is substantial, it often mimics research that is done in cancer centres in HICs rather than reflecting the needs of local patients. In the past 10 years, LMICs have seen an explosion of private cancer centres that have been developed in a mostly unregulated manner. ${ }^{36}$ Deficits in public funding and the absence of a clear national vision and strategy, including failures to develop, fund, and deliver on health and cancer planning, have created a vacuum in public health care that has been rapidly filled by the private sector. Even in countries with traditionally strong public funding, such as Chile $\mathrm{e}^{37}$ and Mexico, ${ }^{38}$ deficits in funding have caused public cancer centres to fall behind in terms of infrastructure investment. This situation has both driven and perpetuated the move of patients, health-care professionals, and resources to private sector cancer centres. Furthermore, cancer centre development in LMICs is driven by a very different set of agendas, contexts, and political motivations leading to ad hoc, often irrational, expenditure on technologies (especially medicines) and projects with no effect on outcomes (eg, prostate-specific antigen screening in lieu of rational, phased development of cancer centre capacity and capability).

The different levels of cancer centres, which have usually been defined in high-income settings, are also subject to substantial heterogeneity in LMICs, as few countries have clear typologies, standards, and workloads within a formal accreditation system. LMICs have recognised the importance of a coherent strategy for developing cancer centres for national and global cancer control and are active in this area by building on preexisting internationally recognised cancer centres that deliver care, training, and research to international standards. Although emerging powers and low-income countries can be grouped together, the hugely heterogeneous nature of these countries, their health ecosystems, and historical contingencies should be recognised.

\section{Asia}

Asia has seen important developments in the expansion of cancer centres and access to care. The National Cancer Institute in Bangkok has a major influence on the development of cancer centres in Thailand and cancer units in several tertiary care hospitals, including university hospitals. Indonesia and the Philippines are still falling behind, with only a few cancer centres developed and long waiting lists for treatment, which is often compounded by their complex geography that includes thousands of islands. Bangladesh and Sri Lanka have made progress in the development of cancer centres. ${ }^{39}$ However, the pace of development has been dependent not just on national commitment to such projects, but also on geography-countries with numerous small island populations and other natural barriers experience serious challenges during the development of a cancer centre network.

In India, the development of cancer centres has been heavily influenced by sociodemographics; $70 \%$ of the population lives rurally but most cancer health-care professionals work in urban areas and nine out of ten of these professionals work in private sector cancer centres because of improved facilities and remuneration, and because of minimal accountability. ${ }^{40}$ Internationally recognised comprehensive cancer centres with substantial research portfolios can be found in major cities such as Delhi (All India Institute of Medical Sciences [AIIMS]), Mumbai (TMC), Kolkata (TMC), Chennai (Women's Indian Association), Bengaluru (Kidwai Memorial), and Ahmedabad (Gujarat Cancer Research Institute). However, these centres face huge clinical volumes not seen in cancer centres in HICs (eg, 42000 patients were treated by 188 staff in TMC Mumbai compared with 41000 patients treated by 1700 staff at MD Anderson Cancer Centre, University of Texas [Pramesh CS, unpublished]), leading to waiting times for diagnosis and treatment that can be in excess of 12 weeks. Despite this situation, public sector cancer centres have delivered research programmes relevant to the Indian population. ${ }^{41}$ Additionally, 29 regional cancer centres have been established by the government of India under the National Cancer Control Programme (NCCP) since 1975. ${ }^{36}$

The uneven development of cancer centres in India is linked to an absence of leadership, funding, and commitment. Successes have included the unique model of TMC Rural Extension Project Nargis Dutt Memorial Cancer Hospital in Barshi, India, extending cancer treatment in rural and impoverished areas, which is a good example of coordination of a hub and spoke model in an LMIC, with TMC Mumbai acting as the hub (for complex care) and the cancer centre in Barshi being one of the spokes for cancer treatment. ${ }^{42}$ This project is a unique experiment in cancer centre development and has relevance for other transitioning countries to develop one major cancer centre as the hub that drives the development of other centres to deliver cancer care 
across the country. However, the BIMARU states of India (Bihar, Madhya Pradesh, Rajasthan, and Uttar Pradesh) have not seen successful cancer centre development possibly as a result of lower literacy rates, slower economic growth, and a higher corruption index than other regions of India.

Although efforts have been made to develop comprehensive cancer centres, unless there is governance on the quality of care with adherence to guidelines, these new cancer centres are not likely to improve patient outcomes. The Indian Council of Medical Research and the National Cancer Grid of India have made efforts t o publish guidelines and consensus statements for common cancers that can be used for quality improvement. ${ }^{17,43}$

\section{Africa}

In Africa, important strides have been made in cancer centre development in Algeria, Egypt, Ghana, Morocco, South Africa, Tanzania, and Tunisia. The National Cancer Institute in Cairo and the Moroccan cancer centre have been major catalysts for human resources in Africa. Morocco has 11 public sector cancer centres and is an exemplar of cancer centre development in Africa, especially in supporting human resource development in Francophone African countries such as Burkina Faso, Chad, Gabon, Mauritania, Niger, and Senegal. ${ }^{44}$ Tunisia provides cancer services through a comprehensive cancer centre in Tunis and cancer units in 24 university hospitals. However, Algeria, Egypt, Morocco, South Africa, and Tunisia account for $80 \%$ of all cancer centres and cancer treatment facilities in Africa. Large and populous African countries such as Democratic Republic of the Congo, Nigeria, and all Francophone African countries have substantially underdeveloped cancer centres. After the end of civil war in Angola in 2002, the government has invested in a modern cancer centre (National Oncology Centre) in Luanda, with all staff trained in Brazil, which was effective for building human r esources into cancer centre development. However, sustaining these positive developments has proved extraordinarily challenging in the face of shifting political commitments and economic priorities. ${ }^{45}$

In Zambia, a major challenge for effective cancer control has been insufficient human resources in surgical oncology for urban cancer centres. To meet this need, the Zambia's National Cancer Control Strategic Plan (2016-21) called for the development of in-country surgical oncology training in tertiary cancer centres. The focus of cancer centre development has been on building up the workforce capacity and capability for women's cancers, facilitated by international collaborations with surgical oncologists from HICs. ${ }^{46}$ Once trained, surgical subspecialists are placed in rural cancer centres in which they offer standard surgical oncology services while serving as leaders for the development of these rural cancer centres. A major academic hospital, the University Teaching Women and Newborn Hospital, recently created a cancer centre by establishing a Gynaecologic Oncology Unit to improve the organisation and delivery of gynaecological cancer care. This development allowed the academic hospital and the newly created site-specific cancer centre to be approved as a site for the International Gynaecologic Cancer Society's Global Training Program. Zambia has thus used the creation of cancer centres for a specific strategically driven focus around surgical oncology workforce. This focus is developed further with various research twinning grants such as the UK Medical Research Council grant to investigate the use of virtual surgical simulation-enhanced training and teleproctoring to enhance the transfer of surgical skills.

Like Zambia, Kenya has experimented with various public and private cancer centres over the past decade that have organically developed in response to need (Kenyan Network of Cancer Organisations). ${ }^{47}$ This experimentation has included novel models based on the dual strategy of twinning and building on existing clinical care infrastructure. AMPATH was initially coined for the Academic Model for the Prevention and Treatment of HIV/AIDS and was created in 2001, to address training issues in Kenya. ${ }^{31}$ The project started as an in-house training programme for health-care professionals to be trained in oncology to bridge a gap in the workforce in Kenya through international collaboration with cancer centres within the Moi University School of Medicine, the Moi Teaching and Referral Hospital, and a consortium of North American academic cancer centres led by Indiana University, USA. ${ }^{31}$ A key driver for this cancer centre development and international collaboration was the need to develop a programme for the management and care of around 50000 previously screened women. Additionally, this cancer centre model was crucial for the expansion into wider oncology treatment. Around 8000 patients are now seen in medical and paediatric haematology and oncology clinics at AMPATH. Financial sustainability has been the major challenge, an issue seen in many LMIC cancer centres. ${ }^{48}$ For the AMPATH model in Kenya, philanthropic donations helped to meet costs, especially for drugs. Additionally, the Ministry of Health and the Kenyan Government have modified the National Hospital Insurance Fund to cover chemotherapy and radiotherapy. One issue has been the comprehensiveness of the medical insurance that covers Kenyan civil servants and their families, which is subsidised by taxpayers' money, meaning that a proportionally greater amount of funding comes from people on lower incomes than those on higher incomes, making this approach hugely regressive. Philanthropy has allowed the Kenyian Chandaria Cancer and Chronic Care Centre to become a cancer centre, with capacity for around 10000 patients as well as for cancer research and education.

\section{Middle East}

Cancer centre development in the Middle East has been greatly challenged by ongoing military and political 
conflicts. For many patients with cancer in Iraq and Syria, for example, the dismantling of previously robust healthcare infrastructure and cancer centres has led to regional patient travel in pursuit of reliable care. ${ }^{49}$ This movement has contributed to the rise of cancer centre hubs that aim to provide services, usually at a considerable cost, to migrants and local populations. ${ }^{50}$ As such, shortcomings in the provision of cancer care and cancer centres in the Middle East need to be understood as a regional rather than a national problem, with one of the fundamental barriers to coordinated cancer control being difficulties in financing care when state funding has been unsustainable and unreliable. Numerous examples of individual pioneers such as the King Hussein Cancer Centre (KHCC), Cancer Institute of Iran, Tehran University of Medical Sciences, and the Kuwait Cancer Control Center, can be found in the development of cancer centres across the Middle East, such as in Iran and Kuwait, but the challenging and changing therapeutic geographies of these health systems impacted by long-term conflict shows how important context and history are in the realistic planning of cancer centre development. The trajectory taken by the KHCC in Jordan is one example of how cancer care is being transformed under the pressures of mass population movements across the region and the effects of war and conflict.

The KHCC, a non-governmental and non-profit institution, was founded in 1997 in response to the fragmentation of cancer care delivery nationwide. ${ }^{51} \mathrm{KHCC}$ has developed into one of the region's leading comprehensive cancer centres, treating around $60 \%$ of national cancer cases, and offering palliative and psychosocial care as part of the cancer care continuum. ${ }^{52}$ The success in scaling up cancer care services at KHCC have largely been attributed to strategic planning, which has included linkage of cancer care provision to medical training, residency programmes, emphasising improvements in quality control, and support from external bodies.

Another element affecting cancer care provision at KHCC, and elsewhere in the region, is the rising number of medical travellers from countries of conflict such as Iraq, Sudan, Syria, and Yemen. The cost of cancer treatment has been kept low-a fraction of the cost in more recently developed health-care centres in Beirutand alternative non-profit insurance programmes funded by annual premiums allow comprehensive treatment of patients regardless of nationality. KHCC development also highlights the importance of external support for national strategies, which can add substantial value if used appropriately. The WHO made Jordan a demonstrative project for palliative care ${ }^{52}$ and was the recipient of a transformational leadership title and support from the US National Cancer Institute.

The movement of populations in pursuit of health care across the region, particularly for cancer for which treatment is time-intensive and expensive, has local implications for national cancer care delivery. Across the
Middle East, many health-care hubs are increasingly tailoring health-care services to medical travellers, providing assistance for paperwork and visa renewals, as well as accommodation. Many cancer centres in this region risk undermining access to cancer treatment at local levels because they focus on meeting regional demands. The effect of patient demographics, particularly on countries hosting refugees, should also not be underestimated. Cancer centre development in the region has been expected to rapidly increase capacity despite almost no international support. ${ }^{53}$

\section{Latin America}

The Brazilian National Cancer Institute and the Porto Alegre University Hospital have played a major part in developing cancer centres and generating vitally needed trained human resources for the Lusaphone African countries such as Angola, Guinea Bissau, and Mozambique. Since 2010, patients with cancer in Brazil have the right to be treated in a comprehensive cancer centre. Despite governmental efforts to certify new centres, only three out of 26 states have enough medium and high-level cancer centres (defined as one centre per 500000 people), including provision of radiotherapy. A national plan was created in 2012 to implement 80 new radiotherapy units. With this effort, nine of the states are expected to achieve adequate radiotherapy coverage for patients treated in the public sector. ${ }^{54}$

Brazil has 43 comprehensive cancer centres and 249 cancer centres, most of which are in states in the south and on the east coast of the country. Patients in Brazil can travel huge distances to be treated at cancer centres, which can affect treatment compliance and cause delays in initiating treatment. According to a national law, all Brazilian citizens with cancer should receive their first treatment within 60 days of a pathology diagnosis of cancer, but this is challenging to deliver. An absence of centralisation into comprehensive cancer centres has led to workforce issues, especially in surgery. Despite breast reconstruction surgery being a civil right since 1999, up to $60 \%$ of patients with breast cancer do not have access to reconstructive surgery as a result of a scarcity of trained reconstructive surgeons who are too spread out amongst the different cancer centres. However, despite this problem, breast reconstruction has increased from 15\% in 2008 to $29 \%$ in $2014 .{ }^{55}$ Compounding challenges to cancer centre development in Brazil include the effect of the economic downturn in 2014, which has meant that since 20152 million Brazilians have lost their health benefit. According to a National Health Supplementary Agency policy published in 2016, this vacuum in public sector funding and in cancer centres will be fulfilled by the private sector, further driving inequalities and catastrophic expenditures on cancer. Crucially, the scarcity of data on the mapping, volumes, and quality of cancer care in cancer centres is a major obstacle to creating evidenced-based policy. 
The development of cancer centres in LMICs is crucial to the delivery of cancer care and improving outcomes for patients. However, LMICs have more heterogeneous and dynamic environments than HICs including in relation to workforce capacity, unregulated growth of the private sector, a scarcity of public sector investment in cancer centres, radically different migration patterns of patients, discontinuities in pathways of care, and a general absence of national strategies for cancer centre development, governance, sustainable financing, and accreditation (including tiering during which a centre provides some aspects of cancer care to a centre that provides comprehensive cancer care). Despite these challenges, many individual centres, countries, and networks have provided a rich mosaic of models and solutions for cancer centre development. National cancer centre strategies are needed to address inequality, including the public-private schism, distributional inequalities (geographical location of cancer centres to serve the greatest number of patients), and quality of care. Furthermore, although cancer centres are often perceived as centres of excellence in HICs, the converse is true in LMICs where they are perceived as places to die. Changing perceptions as well as the stage of cancer at which patients present with disease will be essential for cancer centre development in these radically different sociocultural settings.

\section{Integration and distribution of cancer centres within health systems}

Our analysis and exemplars of cancer centres described herein have discussed some of the challenges that cancer centre development in LMICs face. Policy makers have begun to address how to deliver equality in cancer care and national outcomes through a managed tiered approach to cancer centre development, including improved integration between individual cancer centres to deliver peer review, common guidelines, training, and research networks. ${ }^{56}$

\section{Changes in health policy}

Even in HICs, models of integration are evolving, constantly changing patterns of co-operation and competition in care, and finding new ways to build research networks between cancer centres. Changes to ideology and health policy in HICs have had substantial effects on cancer centre distribution and integrationcare close to home, competition, capacity building, and quality metrics have had both positive and negative effects on outcomes. Patient preference and the provision of

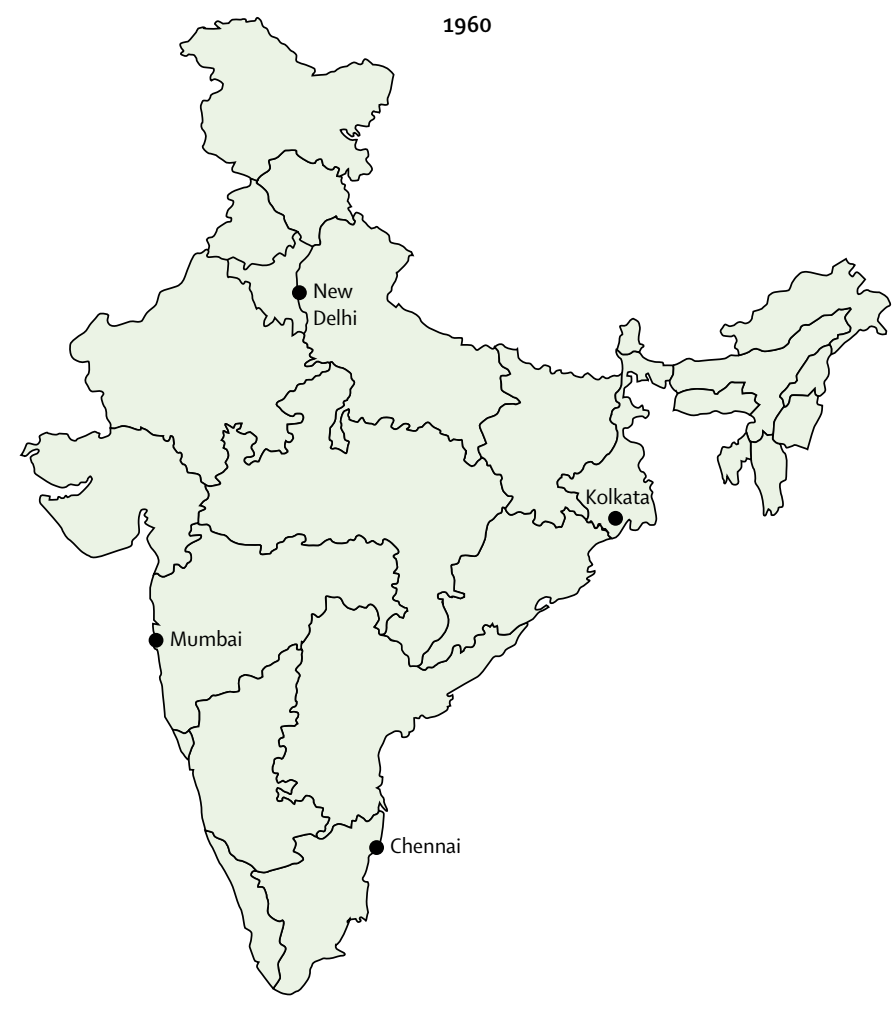

1960

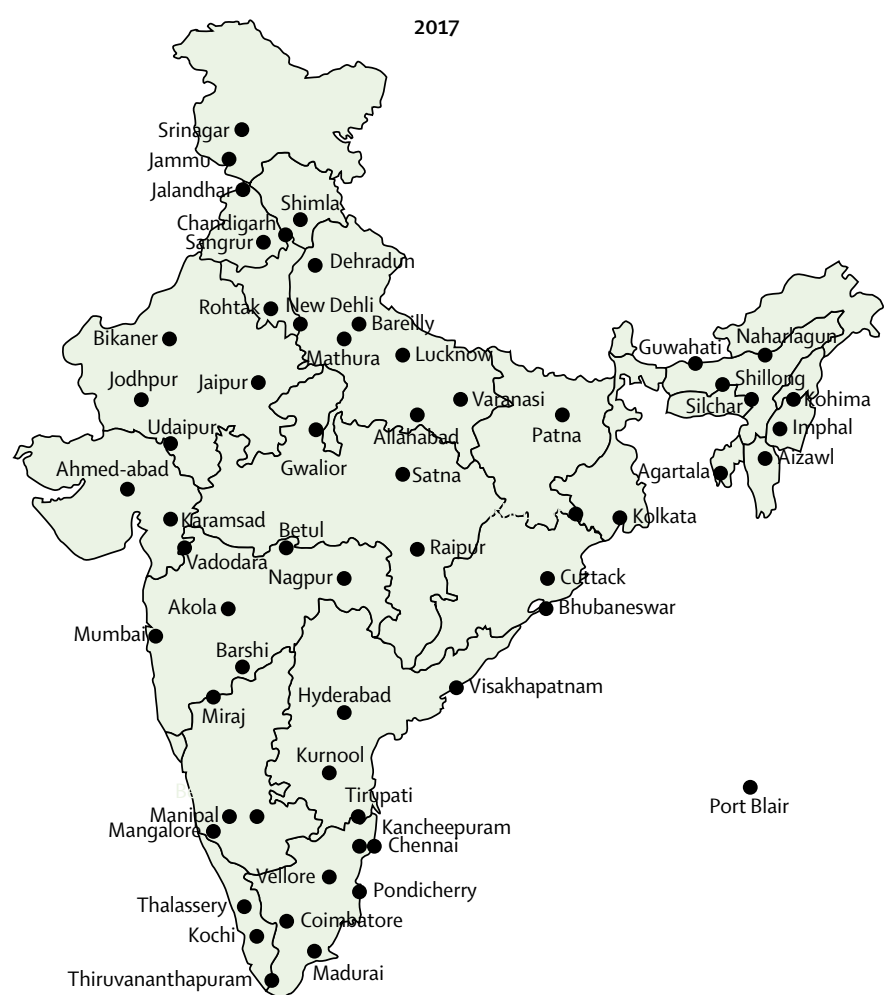

2017

Figure 2: Cancer centres in India that form part of the National Cancer Grid in 2017 compared with cancer centres in 1960

Image is courtesy of C S Pramesh. 


\section{Panel 2: New cancer centre models in India}

Tata Trusts in partnership with the Government of India, are rolling out a step-down distributed cancer centre model, commencing initially in north-east India. The vision is to create patient-centric cancer centres to deliver standardised and affordable care close to patients' homes. The programme has six key factors:

\section{Infrastructure development}

The network will have three levels:

- Level 1: comprehensive cancer centres (around 200 beds) providing services ranging from diagnostics to complex therapies and research

- Level 2: cancer centres (around 100 beds) located near existing government medical colleges offering common diagnostic and treatment services with complex care being delivered at level 1

- Level 3: stand-alone day care cancer units near district hospitals offering diagnostics, chemotherapy, and radiotherapy

\section{Awareness, prevention, and early detection}

Health communication, prevention, and screening programmes for early detection

\section{Community and hospital palliative care}

Integration of clinical guidelines and training

Through the National Cancer Grid of India

\section{Human resource development}

Up-skilling and para-skilling the health-care workforce

\section{Technology to link centres}

Implementation of a Digital Nerve Centre to ensure seamless flow of patient information across centres. All the centres will be linked through teleradiology, telepathology, and virtual tumour boards explosion of private cancer centres. In response, the National Cancer Grid of India has established a network of cancer centres across the country (figure 2) and has been a part of the development of new kinds of cancer centres to serve geographically isolated patient populations (eg, Assam in northeast India; panel 2; figure 3).

Outside country-specific initiatives to integrate cancer centres, notable models have been developed to bring together cancer centres with a site-specific and typespecific focus-eg, breast cancer in Rwanda. ${ }^{59}$ With the considerable reduction of childhood communicable disease mortality over the past two decades in many LMICs, an expected increase in the incidence of childhood cancers (to that of HICs in the 1960s) has occurred. ${ }^{60}$ Many LMICs have created paediatric cancer centres to diagnose and treat children with cancer, often with the help of international twinning partners. ${ }^{33}$ Singlehub cancer centres in LMICs see only $25-30 \%$ of the expected number of cases for each country, with Ghana being a notable example of a twinning partnership between Korle Bu Teaching Hospital, Accra and the Royal Hospital for Sick Children, Edinburgh, UK. ${ }^{61}$ This effect is due, in part, to the distances that patients have to travel to cancer centres, which are usually in capital cities. To overcome these challenges and optimise access for all children, efforts are being made to create networks of childhood cancer centres at the unit level with a hub or referral centre and shared care units spread across the country to match populated areas. Challenges have included recruitment of staff to rural cancer centres and the general issue of proper oversight and referral pathways because most cancer centres operate in an autonomous manner. For adults, cancer centres have been linked in LMICs through research, particularly clinical trial networks. However, most of these networks have been developed as a result of research funding from HICs and few networks in LMICs exist. ${ }^{62}$ The networks that do exist have managed to tie together both research and improved care as strategic drivers to bring cancer centres in different locations together. The Breast Health Global Initiative has brought together cancer centres to work on a resource stratification framework in which breast cancer management strategies can be prioritised within the context of available health-care resources in fulfilment of the 2017 World Health Assembly Cancer Resolution to "adapt stepwise and resource-stratified guidance and tool kits" for comprehensive cancer prevention and control programs..$^{63,64}$

In many LMICs, patient migration in search of care had a profound effect on cancer centre development. The location of 75000 patients visiting the Tata Memorial Centre in Mumbai was mapped and showed that most patients travelled thousands of kilometres to get access to cancer treatment. This effect is also likely to be seen for other major comprehensive cancer centres in India, reflecting the scarcity of adequate cancer care facilities close to peoples' homes.

Such migration has caused massive financial strain on public cancer centres and has led to an unregulated

\section{Focus on quality of care}

Initial cancer centre development in HICs and early adopters of cancer centres such as India show that highvalue care can be delivered by well-structured and organised national cancer centres that deliver geographical and socioeconomic equality. Achieving this goal requires political and clinical leadership with strong patient advocacy. Our analysis shows, however, that 
multiple pitfalls can occur that should be addressed. Many countries provide a wealth of models for cancer centres that have strengths and limitations; however, little structural funding exists to link different cancer centres together. Aligning political focus to support both national and international connections between institutions is essential considering the complexity of rapidly changing therapeutic geographies, and the fact that countries and regions are at very different stages of the cancer transition. ${ }^{65}$ Clinical leadership in cancer centres belong to heterogeneous coalitions with different degrees of power over national decision making and agenda setting. Developing networks of cancer centres requires an integrated approach to capacity and capability rather than a focus on surgical planning ${ }^{66}$ radiotherapy infrastructure, ${ }^{67}$ or generic discussions around strengthening cancer systems. The popular strategy for cancer policy has been to treat these modalities and themes as special areas, yet the central aspect of the structures and organisation through which to deliver pathways and models of care in an equitable manner has been largely ignored. Pronounced challenges exist for co-ordinated multidisciplinary care and patient navigation within LMICs. To provide effective multidisciplinary treatment, focus should also shift towards data entry and maintenance of patient records. If medical records are disorganised or inaccessible, delivering coordinated care or doing research is not possible. Universal health coverage also provides the imprimatur for an analytical policy approach to public and private cancer centres, particularly for private cancer centres in which an absence of governance around structures, quality, outcomes, and geographical location has created huge systemic issues in many countries. ${ }^{68}$

\section{Delivering cancer care in complex settings with competing health priorities}

The social, economic, and political diversity both between and within countries has shaped the way in which cancer centres have developed. Challenges well beyond cancer, including the double and triple burden of disease in most LMICs, have shaped unique experiments in the structure and organisation of cancer centres. Likewise, the effect of the promotion of medical tourism through globalisation, and a focus on deregulation and privatisation, has damaged much needed progress on the public provision of cancer centres for many LMICs in the past two decades. ${ }^{69}$ However, despite the many challenges and setbacks that have been faced, a range of models and expertise are available internationally for the creation of cancer centres that promote the delivery of affordable, equitable, and high-quality care, as well as training and research within NCCPs. Crucially, cancer centres need clear public sector strategic direction and leadership that is built on local expertise but with an understanding of international context, by use of the best evidence base to create sound national policies for developing the

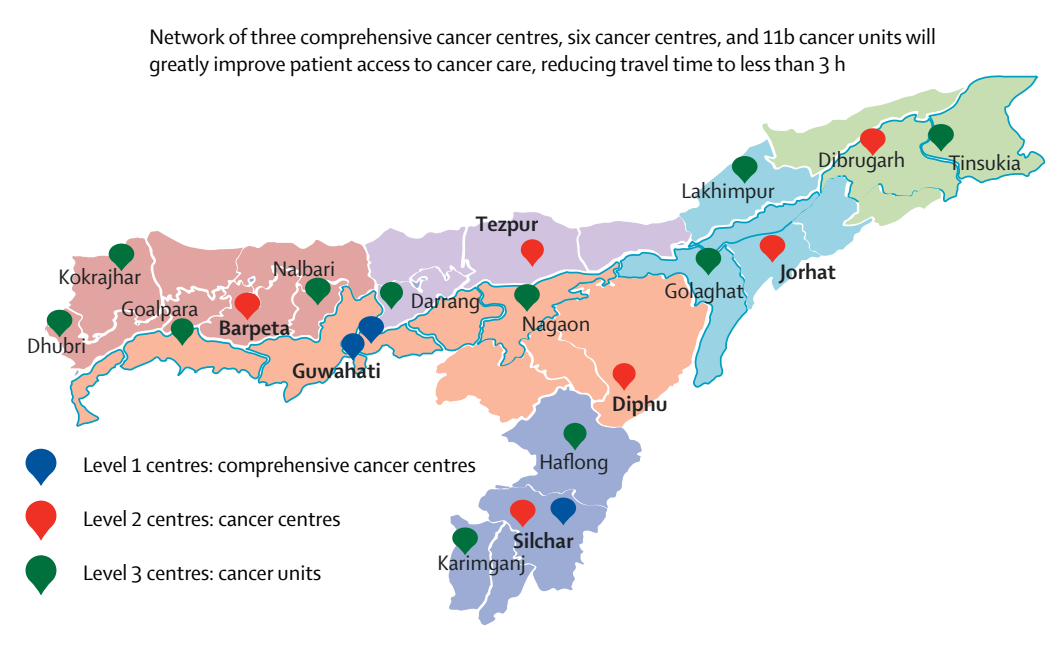

Figure 3: Developing and networking cancer centres in Assam, India Image is courtesy of Arnie Purushotham.

infrastructure and human resources needed for that context. $^{70}$ The reliance on ad-hoc review missions is insufficient for the long-term planning and commitment needed for a national network of public cancer centres. In this health policy analysis, a number of crucial issues and solutions emerge for global cancer centre development.

\section{Health financing models}

The paths for cancer towards Sustainable Development Goal 3 and universal health coverage will require, in addition to political commitment and technological innovation, substantial investment from governments and organisations. ${ }^{71}$ However, public monies alone are unlikely to be enough to achieve these targets. Innovative finance instruments and institutions are becoming increasingly relevant as alternative and sustainable sources of funding..$^{72}$ The UK's Department for International Development was the first bilateral institution to back an innovative model of mixed public and private funding - a development impact bond for education in Rajasthan, India. 3 years on, the education development impact bond is reporting promising results. How would a bond for cancer centre development work? Impact investors would provide start-up or growth capital for the setting up of a cancer centre and implementing organisations would use that capital to deliver cancer care services and possibly prevention services, depending on the structure of the investment and incentive, to a target population. The outcome funders (foundations, bilateral organisations, development banks, national governments, and insurance funds of emerging markets) would repay the investors their principal plus some extra amount if the targets are reached. Irrespective of the chosen instruments, transformative financing for cancer centres is needed to improve the standards of existing centres and to create new ones. ${ }^{73}$ 


\section{Search strategy and selection criteria}

We searched MEDLINE; Web of Science; Library, Information Science and Technology Abstracts; and PubMed using the subject heading and abstract terms, "India", "Latin America", "Africa", "Middle-East", "China", "Low and middle income countries", "comprehensive cancer centre", "cancer centre", "healthcare". Articles published between Jan 1, 1980, and Dec 31, 2017, were included. We also reviewed resource stratified guidelines and Lancet commissions that dealt with cancer care delivery and provision in low-income and middleincome countries.

\section{Role of charities and patient advocacy groups}

An unfortunate aspect of promoting cancer development in LMICs has been the absence of pressure from civil society for both the provision of universal health coverage and the push for increased biomedical research. $^{74}$ In HICs, patients, families, and advocacy agencies have played a major part in stimulating cancer research and pushing for cancer treatments. Having said this, efforts have been made in LMICs to provide end of life care and palliation for patients with cancer and other diseases (eg, Hospice Uganda, CanSupport, India, and Pallium India, although more attention to this issue is needed).

\section{Conclusion}

Modern affordable and equitable cancer care and research demands the integration and fair distribution of cancer centres. However, our analysis of both HICs and LMICs shows that substantial challenges need to be addressed, including ensuring equitability between private and public cancer centres, distributional barriers due to extreme geography or lower development, the scarcity of trained health-care professionals, and distributional inequality due to the coalescing of cancer centres in the same, often urban, areas. Novel solutions do exist, but for these to be effective an alignment of political and cancer centre leadership needs to occur, with incentives for co-operation and the removal of competitive market strategies. ${ }^{75}$ LMICs need to develop models for cancer centres that take into account the substantial mistakes made by HICs. Crucially, this development should be within the context of national planning for health systems, health human resources, and laboratory infrastructure, as well as rational and phased national cancer control planning underpinned by public total health-care expenditure.

\section{Contributors}

BS and RS conceived the content of the paper and drafted the initial manuscript with KC. CSP created figure 2; AP created figure 3. All other authors contributed equally to the writing of the Review and approved the final version.

\section{Declaration of interests}

We declare no competing interests.
Acknowledgments

RS, KC, OED, and OS wish to thank Research Council's UK R4HC-MENA for funding (grant number ES/P010962/1).

\section{References}

1 Sullivan R, Peppercorn J, Sikora K, et al. Delivering affordable cancer care in high-income countries. Lancet Oncol 2011; 12: 933-80.

2 Ginsburg O, Bray F, Coleman MP, et al. The global burden of women's cancers: a grand challenge in global health. Lancet 2017; 389: 847-60

3 Sullivan R, Alatise OI, Anderson BO, et al. Global cancer surgery: delivering safe, affordable, and timely cancer surgery. Lancet Oncol 2015; 16: 1193-224.

4 Atun R, Jaffray DA, Barton MB, et al. Expanding global access to radiotherapy. Lancet Oncol 2015; 16: 1153-86.

5 Lingwood RJ, Boyle P, Milburn A, et al. The challenge of cancer control in Africa. Nat Rev Cancer 2008; 8: 398-403.

6 Balarajan Y, Selvaraj S, Subramanian SV. Health care and equity in India. Lancet 2011; 377: 505-15.

7 Goss PE, Lee BL, Badovinac-Crnjevic T, et al. Planning cancer control in Latin America and the Caribbean. Lancet Oncol 2013; 14: 391-436.

8 Jamison DT, Summers LH, Alleyne G, et al. Global health 2035 a world converging within a generation. Lancet 2013; 382: 1898-955.

9 Stringhini S, Carmeli C, Jokela M, et al. Socioeconomic status and the $25 \times 25$ risk factors as determinants of premature mortality: a multicohort study and meta-analysis of 1.7 million men and women. Lancet 2017; 389: 1229-37.

10 Parham GP, Mwanahamuntu MH, Kapambwe S, et al. Population-level scale-up of cervical cancer prevention services in a low-resource setting: development, implementation, and evaluation of the cervical cancer prevention program in Zambia. PLoS One 2015; 10: e0122169.

11 Howitt P, Darzi A, Yang GZ, et al. Technologies for global health. Lancet 2012; 380: 507-35.

12 Hafner T, Shiffman J. The emergence of global attention to health systems strengthening. Health Policy Plan 2013; 28: 41-50.

13 Ramlogan R, Mina A, Tampubolon G, Metcalfe JS. Networks of knowledge: the distributed nature of medical innovation. Scientometrics 2007; 70: 459-89.

14 Bynum WF, Hardy A, Jacyna S, Lawrence C, Tansey EM. The western medical tradition: 1800 to 2000. Cambridge: Cambridge University Press, 2006.

15 National Cancer Institute. National Cancer Act of 1971. 2016. https://www.cancer.gov/about-nci/legislative/history/nationalcancer-act-1971 (accessed March 14, 2018).

16 Saghatchian M, Thonon F, Boomsma F, et al. Pioneering quality assessment in European cancer centers: a data analysis of the organization for European cancer institutes accreditation and designation program. J Oncol Pract 2014; 10: e342-49.

17 Pramesh CS, Badwe RA, Sinha RK. The national cancer grid of India. Indian J Med Paediatr Oncol 2014; 35: 226-27.

18 Birkmeyer JD, Siewers AE, Finlayson EV, et al. Hospital volume and surgical mortality in the United States. N Engl J Med 2002; 346: $1128-37$

19 Cambrosio A, Keating P, Mercier S, Lewison G, Mogoutov A. Mapping the emergence and development of translational cancer research. Eur J Cancer 2006; 42: 3140-48.

20 Hata T, Takahashi H, Sakai D, et al. Neoadjuvant CapeOx therapy followed by sphincter-preserving surgery for lower rectal cancer. Surg Today 2017; 47: 1372-77.

21 Del Mastro L, De Placido S, Bruzzi P et al. Fluorouracil and dose-dense chemotherapy in adjuvant treatment of patients with early-stage breast cancer: an open-label, $2 \times 2$ factorial, randomised phase 3 trial. Lancet 2015; 385: 1863-72.

22 Dobrow MJ, Goel V, Upshur REG. Evidence-based health policy: context and utilisation. Social Sci Med 2004; 58: 207-17

23 Kingham TP, Alatise OI. Establishing translational and clinical cancer research collaborations between high- and low-income countries. Ann Surg Oncol 2015; 22: 741-46.

24 Ribeiro RC, Antillon F, Pedrosa F, Pui CH. Global pediatric oncology: lessons from partnerships between high-income countries and low- to mid-income countries. J Clin Oncol 2016; 34: 53-61. 
25 Kaito A, Kinoshita T. Educational system of laparoscopic gastrectomy for trainee-how to teach, how to learn. J Vis Surg 2017; 3: 16.

26 Sullivan R, Lewison G, Purushotham AD. An analysis of research activity in major UK cancer centres. Eur J Cancer 2011; 47: 536-44.

27 Simone JV. Understanding cancer centers. J Clin Oncol 2002; 20: 4503-07.

28 Sullivan R. Has the US Cancer Centre model been 'successful'? Lessons for the European cancer community. Mol Oncol 2009; 3: 192-203.

29 Ringborg U, de Valeriola D, van Harten W, et al. Improvement of European translational cancer research. Collaboration between comprehensive cancer centers. Tumori 2008; 94: 143-46.

30 Rajan A, Sullivan R, Bakker S, van Harten WH. Critical appraisal of translational research models for suitability in performance assessment of cancer centers. Oncologist 2012; 17: e48-57.

31 Strother RM, Asirwa FC, Busakhala NB, et al. AMPATH-Oncology: a model for comprehensive cancer care in sub-Saharan Africa. J Cancer Policy 2013; 1: e42-e48.

32 Stulac S, Binagwaho A, Tapela NM, et al. Capacity building for oncology programmes in sub-Saharan Africa: the Rwanda experience. Lancet Oncol 2015; 16: e405-13.

33 Hopkins J, Burns E, Eden T. International twinning partnerships: an effective method of improving diagnosis, treatment and care for children with cancer in low-middle income countries. J Cancer Policy 2013; 1: e8-e19.

34 Brennan M. The role of U.S. cancer centers in global cancer care. Ann Surg Oncol 2015; 22: 747-49.

35 Oluoch T, de Keizer N, Langat P, et al. A structured approach to recording AIDS-defining illnesses in Kenya: a SNOMED CT based solution. J Biomed Inform 2015; 56: 387-94.

36 Goss PE, Strasser-Weippl K, Lee-Bychkovsky BL, et al. Challenges to effective cancer control in China, India, and Russia. Lancet Oncol 2014; 15: 489-538.

37 Jimenez de la Jara J, Bastias G, Ferreccio C, et al. A snapshot of cancer in Chile: analytical frameworks for developing a cancer policy. Biol Res 2015; 48: 1-15.

38 Aggarwal A, Unger-Saldana K, Lewison G, Sullivan R. The challenge of cancer in middle-income countries with an ageing population: Mexico as a case study. Ecancermedicalscience 2015; 9: 536.

39 Hussain SA, Sullivan R. Cancer control in Bangladesh. Jpn J Clin Oncol 2013; 43: 1159-69.

40 Mallath MK, Taylor DG, Badwe RA, et al. The growing burden of cancer in India: epidemiology and social context. Lancet Oncol 2014; 15: e205-e12.

41 D'Cruz AK, Vaish R, Kapre N, et al. Elective versus therapeutic neck dissection in node-negative oral cancer. N Engl J Med 2015; 373: 521-29.

42 Desai S, Patil R, Kothari A, et al. Static telepathology consultation service between Tata Memorial Centre, Mumbai and Nargis Dutt Memorial Charitable Hospital, Barshi, Solapur, Maharashtra: an analysis of the first 100 cases. Indian J Pathol Microbiol 2004; 47: 480-85.

43 Shukla HS, Sirohi B, Behari A, et al. Indian Council of Medical Research consensus document for the management of gall bladder cancer. Indian J Med Paediatr Oncol 2015; 36: 79-84.

44 Selmouni F, Zidouh A, Belakhel L, et al. Tackling cancer burden in low-income and middle-income countries: Morocco as an exemplar. Lancet Oncol 2018; 19: e93-e101.

45 Armando A, Bozzetti MC, de Medeiros Zelmanowicz A, Miguel F. The epidemiology of cancer in Angola-results from the cancer registry of the national oncology centre of Luanda, Angola. Ecancermedicalscience 2015; 9: 510.

46 Chibwesha C, Pinder LF, Musonda A, et al. A comprehensive assessment of breast and cervical cancer control infrastructure in Zambia. J Cancer Policy 2017; 13: 24-29.

47 Topazian H, Cira M, Dawsey SM, et al. Joining forces to overcome cancer: the Kenya cancer research and control stakeholder program. J Cancer Policy 2016; 7: 36-41.

48 Strother RM, Asirwa FC, Busakhala NB, et al. The evolution of comprehensive cancer care in Western Kenya. J Cancer Policy 2013; 1: e25-e30.

49 Dewachi O, Skelton M, Nguyen VK, et al. Changing therapeutic geographies of the Iraqi and Syrian wars. Lancet 2014; 383: 449-57.
50 Dewachi O, Rizk A, Singh NV. (Dis)connectivities in wartime: the therapeutic geographies of Iraqi healthcare-seeking in Lebanon. Glob Public Health 2018; 13: 288-97.

51 Abdel-Razeq H, Attiga F, Mansour A. Cancer care in Jordan. Hematol Oncol Stem Cell Ther 2015; 8: 64-70.

52 Stjernsward J, Ferris FD, Khleif SN, et al. Jordan palliative care initiative: a WHO Demonstration Project. J Pain Symptom Manage 2007; 33: 628-33.

53 Spiegel P, Khalifa A, Mateen FJ. Cancer in refugees in Jordan and Syria between 2009 and 2012: challenges and the way forward in humanitarian emergencies. Lancet Oncol 2014; 15: e290-97.

54 Strasser-Weippl K, Chavarri-Guerra Y, Villarreal-Garza C, et al. Progress and remaining challenges for cancer control in Latin America and the Caribbean. Lancet Oncol 2015; 16: 1405-38.

55 Freitas-Junior R, Gagliato DM, Moura Filho JWC, et al. Trends in breast cancer surgery at Brazil's public health system.J Surg Oncol 2017; 115: 544-49.

56 Brown BB, Patel C, McInnes E, Mays N, Young J, Haines M. The effectiveness of clinical networks in improving quality of care and patient outcomes: a systematic review of quantitative and qualitative studies. BMC Health Serv Res 2016; 16: 360.

57 Aggarwal A, Lewis D, Sujenthiran A, et al. Determinants of patient mobility for radical prostate cancer radiation therapy in the precision medicine era: a population-based study of choice and competition in England. Int J Radiat Oncol 2017; 99: E388.

58 Aggarwal A, Lewis D, Mason M, Purushotham A, Sullivan R, van der Meulen J. Effect of patient choice and hospital competition on service configuration and technology adoption within cancer surgery: a national, population-based study. Lancet Oncol 2017; 18: 1445-53.

59 Murthy SS, Tapela N, Muhimpundu MA, et al. A national framework for breast cancer control: a report on Rwanda's inaugural symposium on the management of breast cancer J Cancer Policy 2015; 6: 3-7.

60 Howard SC, Metzger ML, Wilimas JA, et al. Childhood cancer epidemiology in low-income countries. Cancer 2008; 112: 461-72.

61 Renner L, Segbefia C, Johnson E, Burns E, Sharma V, Kerr S. Challenges and success in a twinning partnership in Ghana. Oncol News 2013; 8: 20-22.

62 Gonzalez Block MA. The state of international collaboration for health systems research: what do publications tell? Health Res Policy Syst 2006; 4 : 7.

63 Anderson BO, Shyyan R, Eniu A et al. Breast cancer in limited-resource countries: an overview of the Breast Health Global Initiative 2005 guidelines. Breast J 2006; 12 (suppl 1): S3-15.

64 Shen L, Shan YS, Hu HM, et al. Management of gastric cancer in Asia: resource-stratified guidelines. Lancet Oncol 2013; 14: e535-47.

65 Chongsuvivatwong V, Phua KH, Yap MT, et al. Health and health-care systems in southeast Asia: diversity and transitions. Lancet 2011; 377: 429-37.

66 Burssa D, Teshome A, Iverson K, et al. Safe surgery for all: early lessons from implementing a national government-driven surgical plan in Ethiopia. World J Surg 2017; 41: 3038-45.

67 Datta NR, Samiei M, Bodis S. Radiation therapy infrastructure and human resources in low- and middle-income countries: present status and projections for 2020. Int J Radiat Oncol Biol Phys 2014; 90: 971-72.

68 McPake B, Hanson K. Managing the public-private mix to achieve universal health coverage. Lancet 2016; 388: 622-30.

69 Thomson M, Kentikelenis A, Stubbs T. Structural adjustment programmes adversely affect vulnerable populations: a systematic-narrative review of their effect on child and maternal health. Public Health Rev 2017; 38: 13.

70 Liverani M, Hawkins B, Parkhurst JO. Political and institutional influences on the use of evidence in public health policy. A systematic review. PLoS One 2013; 8: e77404.

71 Kenny C. Fifteen years isn't that long: the SDGs and holistic development. 2017. https://www.cgdev.org/blog/fifteen-years-isntlong-sdgs-and-holistic-development (accessed Dec 30, 2017).

72 Atun R, Silva S, Knaul FM. Innovative financing instruments for global health 2002-15: a systematic analysis. Lancet Glob Health 2017; 5: e720-26. 
73 Stenberg K, Hanssen O, Edejer TT, et al. Financing transformative health systems towards achievement of the health Sustainable Development Goals: a model for projected resource needs in 67 low-income and middle-income countries. Lancet Glob Health 2017; 5: e875-87.

74 Pramesh CS, Venkataramanan R, Suvarna V et al. Involvement of general public in biomedical research. Perspect Clin Res 2016; 7: $152-55$.
75 Kumar RV, Bhasker S. Scope, functioning, current problems and future of Peripheral Cancer Centers (PCCs) in India. J Cancer Policy 2016; 9: 18-23.

(C) 2018 Elsevier Ltd. All rights reserved. 